\title{
Wer entspannt ist, nimmt mehr wahr
}

\author{
Intuition spielt in der Heilpraktiker- \\ Patienten-Beziehung eine wichtige \\ Rolle. Sie hilft dem Behandler zu \\ erkennen, was sein Patient benötigt \\ und weist ihm den Weg in der Thera- \\ pie. Doch nur, wenn der Heilpraktiker \\ ganz entspannt ist, kann er zu einer \\ gesteigerten Intuition gelangen, wie \\ eine Forschungsarbeit an der Universi- \\ tät Witten-Herdecke zeigt.
}

Neulich erzählte eine befreundete Heilpraktikerin, sie habe die besten Erfolge in der Behandlung ihrer Patienten, wenn es ihr gelänge, „ganz bei ihnen zu sein“ und sich „Vollkommen auf sie einzulassen“. Es wäre, als könne sie dann „spüren“, was ein Patient gerade benötige und wo sie nach Schwachstellen sowie Potenzialen suchen müsse. Das aber, so sagte sie, gelänge nicht immer. Es ginge nur an Tagen, an denen sie eine tiefe Ruhe in sich spüre und das Gefühl habe, ganz in ihrer Mitte zu sein.

Wie dieser Heilpraktikerin geht es vielen anderen auch bei ihrer Arbeit. Sie greifen selten allein auf ihr fundiertes Wissen in Anatomie, Physiologie, Krankheitslehre und entsprechend der Spezialisierung dann auch auf Homöopathie, TCM etc. zurück. Ebenso wichtig wie ihr objektives Wissen ist für sie die subjektive Wahrnehmung bei Diagnostik und Therapie. Mit ihren 5 Sinnen erhalten diese Heilpraktiker, Ärzte und andere Therapeuten wichtige Informationen von einem Patienten.

Ein Teil dieser subjektiven Wahrnehmung ist die sog. verfeinerte Wahrnehmung oder auch Intuition genannt. Der Heilpraktiker nutzt dabei den subjektiven Eindruck, den er von einem Patienten bekommt, während er mit ihm spricht oder ihn behandelt, für die Therapie.

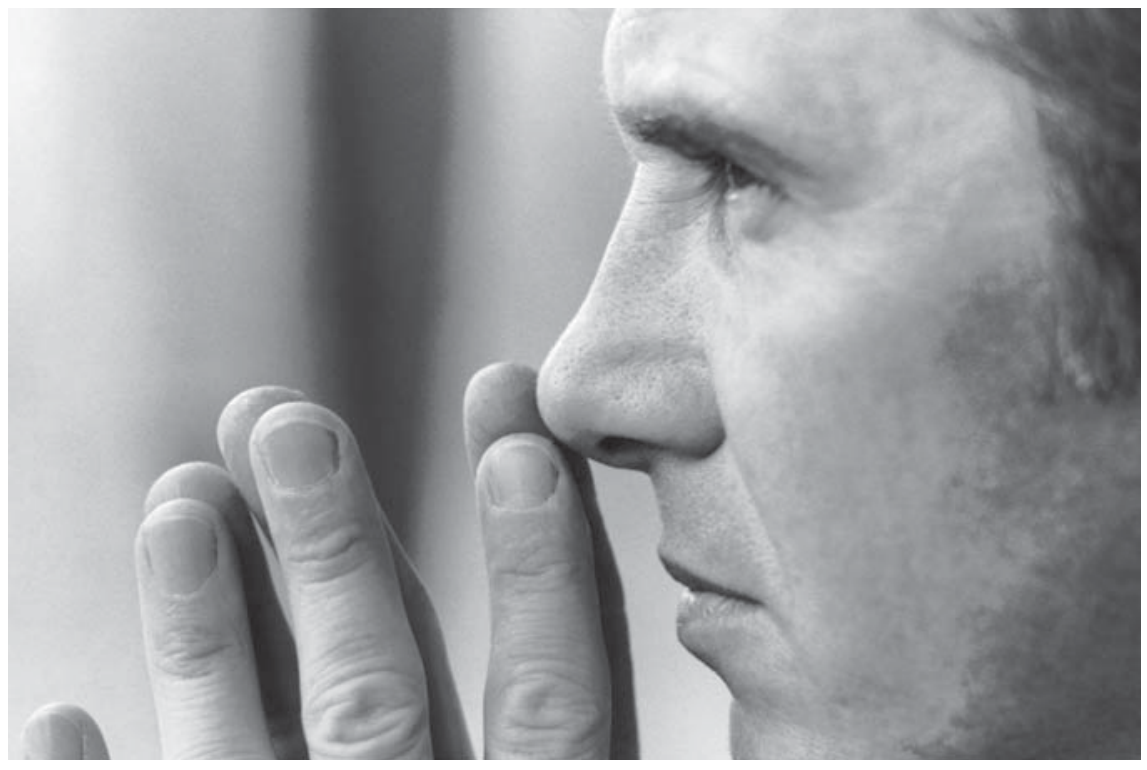

Abb. 1 Ist ein Heilpraktiker entspannt, erweitert sich dadurch seine Wahrnehmung. In diesem Zustand der gesteigerten Intuition erkennt er besser, was sein Patient braucht. Foto: @ PhotoDisc

Intuition in der Heilkunde bedeutet also, ständig das eigene Fachwissen mit empathischer Erkenntnisfähigkeit zu verbinden und die weitere Behandlung darauf abzustimmen. Das klingt zunächst nach einem ganz einfachen Prinzip: Man achtet auf spontan auftretende körperliche und emotionale Empfindungen bei sich und seinem Gegenüber - und schon erhält man eine verbesserte Sicht auf einen Patienten. Doch ganz so einfach wie das klingt, ist es nicht.

\section{Phänomenologisches Wahrnehmen}

Der Zugang zum eigenen Körperbewusstsein und damit zur Intuition setzt eine gewisse Wahrnehmungsqualität voraus - und diese erreicht man wie Untersuchungen zeigen am besten in tiefer Entspannung. Wahrnehmung heißt, seine Achtsamkeit dem Hier und Jetzt zuzuwenden. In einem entspannten Zustand lässt sich auf das, was einem im selben Moment sinnlich begegnet, deutlicher und besser achten. Eine Forschungsarbeit an der Universität WittenHerdecke hat gezeigt, wie sich die Wahrnehmung unter körperlicher und geistiger Entspannung verändert und so die Intuition verstärkt. Die Wissenschaftler haben dazu geprüft, welche Aufmerksamkeitszustände bei dieser besonderen Wahrnehmung auf sinnesphysiologischer und psychologischer Ebene vorliegen. Ihr Ziel war es, den Einfluss des vegetativen und zerebralen Zustands auf die Qualität der Wahrnehmungseindrücke hin zu analysieren.

\section{Untersuchung mithilfe der Medita- tionstafeln von Chartres}

Für die Untersuchung bildeten die Wissenschaftler 2 Gruppen. Die Teilnehmer aus beiden Gruppen unterzogen sich dem- 


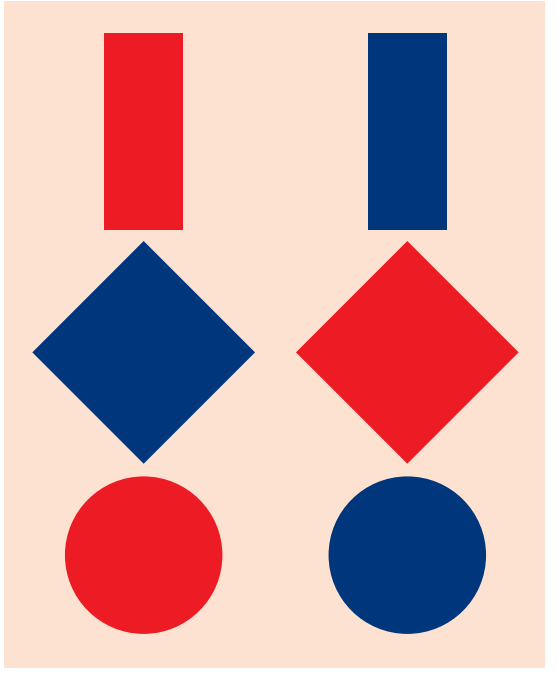

Abb. 2 Die Meditationstafeln von Chartres.

selben Wahrnehmungsversuch, allerdings unter verschiedenen Bedinungen. Die Teilnehmer in der 1. Gruppe entspannten vor dem Wahrnehmungsversuch mit der komplementär-therapeutischen Entspannungsmaßnahme Ergosoma (\$ Kasten). Die Methode diente schon im alten Asien dazu, die Wahrnehmungsfähigkeit zu schulen und zu trainieren. Die Teilnehmer der anderen Gruppe entspannten nicht.

Im Anschluss zeigten die Forscher den Teilnehmern der beiden Gruppen die Meditationstafeln von Chartres ( Abb. 2). Der Anblick der Tafeln mit 2 gleichen Krei- sen unterschiedlicher Farben löst einen sog. binokularen Streit der Augen aus. Der Betrachter sieht für einen Augenblick einen roten, für einen Augenblick einen blauen, dann wieder einen roten Kreis usw. Das liegt daran, dass 2 verschiedene Bilder in jedem Auge wahrgenommen werden, die das Gehirn zu einem Gesamtbild zusammenbringen muss. Vergleichen lässt sich das in etwa mit dem, was wir beim räumlichen Sehen erleben: Der Streit besteht darin, dass die beiden Bilder in den Gehirnarealen so verarbeitet werden, dass sie abwechselnd dominant sind. Beide Bilder können aber auch zu einem verschmelzen. Das ist der Moment, bei dem ein neuer Wahrnehmungseindruck entsteht, ein phänomenologisches Wahrnehmen. Bei den Tafeln von Chartres kann dabei zusätzlich zu den 2 sich ständig streitenden Bildern mit einem roten und einem blauen Kreis der Eindruck von 3 Kreisen entstehen, mit einem violetten in der Mitte (- Abb. 3). Andere aus der Neurowissenschaft bekannte Doppelbilder sind so gewählt, dass ein längs- und quergestreiftes Muster nicht zu einem Bildeindruck fusioniert werden und der Streit so genau beobachtet werden kann. Beim therapeutischen Wahrnehmen kommt es aber auf eine Gesamtsicht an, daher interessiert der Einklang der Bilder, wenn ein neuer Wahrnehmungseindruck entsteht.
Jeder Studienteilnehmer erhielt einen Handdrücker, mit dem er markieren konnte, wann und ob überhaupt für ihn zusätzlich zu dem roten und blauen Kreis der violette sichtbar wurde. Ob er also einen $\mathrm{Zu}-$ stand phänomenologischen Wahrnehmens erreichen konnte.

Im Ergebnis zeigte die Forschungsarbeit: Wesentlich mehr Studienteilnehmer, die zuvor mit Entspannung durch Ergosoma ihre Wahrnehmungsfähigkeiten erhöht hatten, konnten im Versuch vergleichsweise öfter den violetten Kreis zusätzlich zu den beiden anderen erkennen. In der Gruppe ohne Entspannung haben 7 \% das visuelle Phänomen der 3 Kreise mit einer Farbänderung gesehen. In der Gruppe mit Entspannung lag das Ergebnis bei $83 \%$ !

\section{Mehr sinnliche Eindrücke bei vertiefter Wahrnehmung}

Warum das so ist, lässt sich anhand der bei den Untersuchungen vorgenommenen Messungen erklären: Während der Ergosoma-Übungen entspannten sich die Studienteilnehmer aus der 1. Gruppe, was sich im sog. Vegetativportrait als charakterliches Bild zeigte. Sie erreichten einen $\mathrm{Zu}-$ stand, der grundlegend für eine verfeinerte Wahrnehmung ist: Atem- und Herzrhythmus der Teilnehmer wurden durch die vegetative Entspannungsreaktion stabil und

\section{Ergosoma}

Die Ergosoma-Methode ist eine komplementärmedizinische Methode nach R. V. Tajon. Sie hat ihre Wurzeln in den traditionellen Heilmethoden des alten Asiens.

Jeder Mensch hat ein angeborenes Vermögen zur Selbstheilung, Selbstregulierung und Regeneration, einen gesunden, inneren Kern - eine Quelle, aus der alle integrierenden, regulierenden und vitalen Impulse kommen. Daraus ergibt sich der Ansatz, alle Funktionen und Aspekte im Menschen zu fördern, in ihrer Wirkung zu unterstützen und deren Vernetzung anzuregen. Man orientiert sich hierbei an der Salutogenese und nicht am Defizit. Der Behandler entwickelt ein fein ausgeprägtes Palpationsvermö- gen. Er ertastet und erspürt feinste Spannungen, Festigkeiten, Beweglichkeiten, rhythmische Bewegungen wie Pulsation, Ausdehnung und Kontraktion, thermische und energetische Phänomene. Dabei orientiert er sich am Vitalitätsgrad des Gewebes und der energetischen Kraftzentren. Die Einwirkungen bestehen aus zugewandter, neutraler, horchender Aufmerksamkeit und tastenden Berührungen unterschiedlicher adäquater Intensität (z. B. Halten, Drücken, Traktion sowie kreisende und lineare Massagestreichungen). Der Klient reagiert darauf individuell mit Veränderung der Körperspannung (Anspannung, Entspannung), des Atemrhythmus in Frequenz und Tiefe, der Pulsfrequenz und Intensität, der Augenbewegungen (z. B. REM), Änderung der lokalen oder großflächigen thermischen Ausstrahlung und Hautdurchblutung, des Wachzustands der momentanen Aufmerksamkeit, des Muskeltonus u.v.a.m.

Diese Behandlungsform wird seit einigen Jahren in Deutschland, Österreich und der Schweiz ausgebildet und von verschiedenen therapeutischen Berufsgruppen wie Physiotherapeuten, Kranken- und Altenpflegern, Psychotherapeuten, Heilpraktikern etc. und in der Schmerztherapie, Palliativmedizin, Hospizarbeit u. v. m. angewendet.

\section{Informationen zu Ergosoma:}

www.ergosom.ch, www.ergosom-info.de, www.ergosom-graz.at 
regelmäßig, das Nervensystem kam zur Ruhe und die Hirnströme synchronisierten. Dabei vernetzten sich rechte und linke Hirnhälfte miteinander, was die geistige Flexibilität der Teilnehmer steigerte. Sie kamen somit in einen Zustand gesteigerter Aufmerksamkeit - in eine erweiterte Wahrnehmung. Das ermöglichte es ihnen, mehr visuelle Eindrücke während des Wahrnehmungsversuchs zu erleben, als den Teilnehmern aus der 2. Gruppe.

Während sie die Meditationstafeln von Chartres betrachteten, so schilderten die Teilnehmer aus der 1. Gruppe, erlebten sie bei vertiefter Wahrnehmung mehr sinnliche Eindrücke und subjektive Erfahrungen. Auch die Anzahl ihrer erlebten Empfindungen nahm zu. Den Forschern zeigte sich, dass eine vertiefte Wahrnehmung demnach eine spezielle mentale Ausrichtung braucht, damit die Wahrnehmungsspektren zunehmen können.

Bei der Untersuchung fiel jedoch noch etwas auf: Es ließ sich während der vertieften Wahrnehmung mit der entspannenden Ergosoma-Anwendung und dem Schauen im Wahrnehmungsversuch eine veränderte Informationsverarbeitung nachweisen. Was die Teilnehmer der Entspannungsgruppe als ein „zeitfreies Erleben" schilderten, war die Aufhebung des typischen 3-Sekunden-Rhythmus der alltäglichen Informationsverarbeitung. Unser Sinnessystem sucht evolutionär geprägt üblicherweise alle 3 Sekunden nach neuen Informationen bzw. Reizen. Dieser Takt der Sinne hob sich im Moment der erweiterten Wahrnehmung zugunsten eines kontinu-
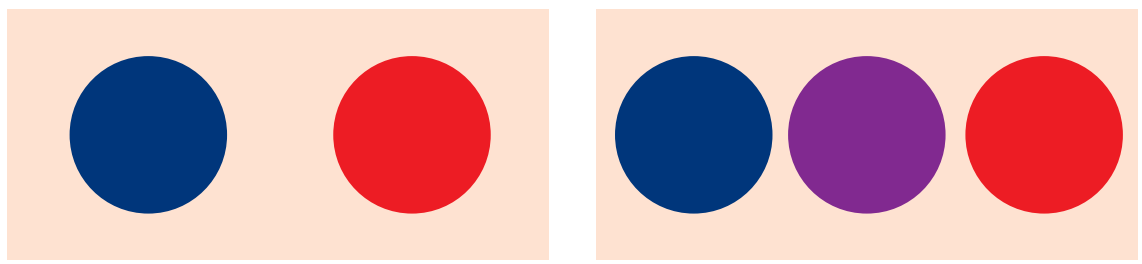

Abb. 3 Aus den beiden sich ständig streitenden Kreisen (li.) kann ein dritter entstehen (re.): ein visuelles Phänomen bei erweiterter Wahrnehmung.

ierlich andauernden Momentes durch einen sog. On-Off-Mechanismus der Gehirnaktivität auf. Für die Forscher dabei besonders interessant: Dies fand in dem Gehirnareal statt, das für die Integration der einzelnen Eindrücke zu einem Gesamtblick oder Überblick des Gesehenen zuständig ist. In diesem Bereich entsteht ein fortwährend fließendes Ereignis über einen längeren Zeitraum.

\section{Diagnosemittel Intuition}

Diese gemessenen geistigen Zustände lassen sich im Sinne der Neurophilosophie als bewusstseinserweiternder Zustand und höhere Denkleistung deuten. Das Entwickeln eines therapeutischen Wahrnehmens formt auch die Denkstruktur des Therapeuten, alleine durch die Ausrichtung seiner Aufmerksamkeit.

Durch das phänomenale Wahrnehmen (griechisch phainomenon „Sichtbares, Erscheinung“; logos „ Rede, Lehre“) erschließt sich einem intuitiv arbeitenden Heilpraktiker ein neuer, subjektiver Gesamteindruck seines Patienten. Dem muss allerdings nicht mehr allein eine logisch-rational erfassbare Erklärung zugrunde liegen.
Bei aller Validierung, Standardisierung und Qualitätsprüfung der Methoden ist auch heute ein phänomenologisch geprägtes Vorgehen eine wichtige Ergänzung, um die Messergebnisse über Prozesse der organischen Systeme und den Zustand des Patienten nachspürend in Diagnose und Behandlung zu ergänzen. Diese Herangehensweise ist aus vielen Traditionen der Volksheilkunde bekannt und kann als Handwerkzeug des therapeutischen Wahrnehmens gut eingesetzt werden. Das therapeutische Wahrnehmen, die Empathiefähigkeit bzw. Intuition, unterstützt den Behandler dabei, mit dem Patienten in Verbindung zu treten, Verständnis für seine Situation zu bekommen und einen objektiven sowie subjektiven Zugang zu ihm und seiner Situation zu finden. Fühlt sich der Patient ernst- bzw. wahrgenommen, gibt dies meist schon eine Entlastung. Die Heilung beginnt dann mit dem Wahrnehmen und der empathischen Wechselbeziehung zwischen Behandler und Patient.

Das Handwerkszeug Intuition kann man trainieren. Es gibt Wahrnehmungsschulungen wie die Kinästhetik, Achtsamkeitsschulungen mit Bewegungsübungen und 
das Körperspüren in der Stille oder mit Musik und Tönen. Diese Übungen können aus allen Richtungen kommen wie dem Kampfsport, aus dem Theater, dem QiGong oder der Meditation. Es gibt dabei Wahrnehmungsübungen, die sich auf den Körper konzentrieren und dort beispielsweise Spannungen, Schmerz, Disbalance, Unruhe oder die Temperatur erspüren. Es gibt auch Wahrnehmungsübungen auf der Gefühlsebene, wobei Angst, Ärger oder Trauer erspürt werden. Ebenso kann eine Übung aber auch im Mentalen liegen und sich auf Wohlgefühl oder Weite konzentrieren.

\section{In der Ruhe liegt die Kraft}

Arbeitet ein Heilpraktiker also mit einer Entspannungsmethode an sich selbst und/ oder mit dem Patienten, kann er eine hohe Aufmerksamkeit bei gleichzeitiger Entspannung erreichen. Ein Zustand von Ruhe und Gelassenheit stellt sich ein, der sich auch dem Patienten vermittelt. Mit einer Entspannungsmethode hat der Behandler eine gute Möglichkeit, seine Empathiefähigkeit zu steigern und damit in eine bessere Verbindung zu kommen, mit sich selbst und mit seinen Patienten.

Die erweiterte Wahrnehmung ist bei allen Therapiemethoden von Vorteil, einschließlich derer der Schulmedizin. Ein weiterer Vorteil für den Heilpraktiker liegt darin, dass er in schwierigen Behandlungssituationen in seiner Souveränkraft bleiben und in Verbindung mit der Intuition Lösungswege erkennen kann. Auch ein eventueller Therapiestillstand kann durch die Impulse aus der empathischen Verbindung wieder in Bewegung kommen. Es ist auch möglich, Ergosoma als alleinige Therapiemethode anzuwenden

Die Herausforderung des Heilpraktikers ist es, die unterschiedlichen Wahrnehmungen zu einem Gesamteindruck zu vereinen, eine sog. Synästhesie zu schaf- fen. Eine erweiterte Wahrnehmung lässt diesen Weit- oder Überblick entstehen, der in einem Moment der Ruhe zur Wirkung kommt. Ist der Heilpraktiker in seinem empathiefähigen Handeln entspannt und wach zugleich, kann er die Informationen über den Patienten, seine eigenen Wahrnehmungen als Resonanz auf die Betrachtung des Patienten klarer erkennen und zugleich die Impulse in gesunder Neutralität und intuitiv an diesen weitergeben.

Eine Grundvoraussetzung für jeden Behandler ist also die innere Stabilität und Ruhe. Ein entspannter Körper, Gelassenheit und klare Gedanken geben Raum für phänomenologisches Wahrnehmen. Das Ziel im Hinblick auf die therapeutische Tätigkeit ist neben der Stabilität und Souveränität ein Zustand von Gewahrsein und Achtsamkeit, um zu erkennen, welche Information der Patient für seine Gesundung benötigt. Gemeinsam kommen beide Seiten durch die verstärkte Empathie in eine Interaktion, in der Entspannung und Harmonie den Weg zur Gesundheit verstärken.

Dieser Artikel ist online zu finden unter: http://dx.doi.org//10.1055/s-0030-1249154

\section{(1) Weiterführende Literatur}

Fuchs T. Das Gehirn - ein Beziehungsorgan. Eine phänomenologisch-ökologische Konzeption. Stuttgart: Kohlhammer; 2008

Klemenz B. Ressourcenorientierte Diagnostik und Intervention bei Kindern und Jugendlichen. Tübingen: DGVT; 2003

Spielberg P. Schul- und Komplementärmedizin: Miteinander statt nebeneinander. Dtsch Ärztebl 2007; 104: A-3148/B-2770/C-2672

v Weizsäcker V. Der Gestaltkreis: Theorie der Einheit von der Wahrnehmung und Bewegung. Stuttgart: Thieme; 1947

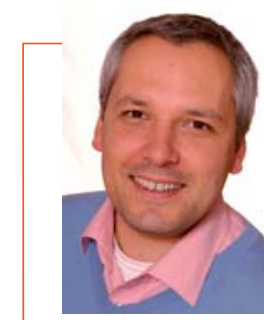

Dr. rer. medic. Markus Köhl Uni. Witten-Herdecke/ ZEPU GmbH, Zentrum für Elektropathologie und Umweltmedizin Stockumer Str. 28, 58453 Witten

Markus Köhl studierte Kunst, Literatur und Medienwissenschaften. Er forschte als Promotionsassistent an der medizinischen Fakultät der Universität Witten-Herdecke im Bereich der Sinnesphysiologie über die Erweiterung der Wahrnehmung, therapeutisches Wahrnehmen und den kreativen Moment der Gesundheit.

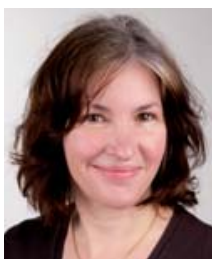

HP Monika Volkmer Zindelstr. 3-6 37073 Göttingen

Monika Volkmer wendet in ihrer Praxis Ergosoma als eigenständige Methode an. Unterstützend setzt sie homöopathische Einzelmittel und Heilpflanzen ein. Freiberuflich arbeitet sie seit vielen Jahren als Ayurvedatherapeutin in der Habichtswaldklinik Ayurveda in Kassel.

E-Mail: monikavolkmer@web.de 